\title{
Generation of diffraction-free plasmonic beams with one-dimensional Bessel profiles
}

\author{
Cesar E. Garcia-Ortiz, ${ }^{1,2, *}$ Victor Coello, ${ }^{3}$ Zhanghua Han, ${ }^{2,4}$ and Sergey I. Bozhevolnyi ${ }^{2}$ \\ ${ }^{1}$ UANL, Facultad de Ciencias Físico-Matemáticas, San Nicolás de los Garza, Nuevo León 66450, Mexico \\ ${ }^{2}$ Institute of Technology and Innovation, University of Southern Denmark, Niels Bohrs Allé 1, 5230 Odense M, Denmark \\ ${ }^{3}$ Centro de Investigación Científica y Educación Superior de Ensenada, PIIT, Apodaca, Nuevo León 66600, Mexico \\ ${ }^{4}$ Center for $\mathrm{THz}$ Research, China Jiliang University, Hangzhou 310018, China \\ *Corresponding author: cegarcia@cicese.mx
}

Received December 19, 2012; accepted February 5, 2013;

posted February 8, 2013 (Doc. ID 182090); published March 12, 2013

\begin{abstract}
We demonstrate experimentally generation of diffraction-free plasmonic beams with zeroth- and first-order Bessel intensity profiles using axicon-like structures fabricated on gold film surfaces and designed to operate at a wavelength of $700 \mathrm{~nm}$. The central beam features a very low divergence $(\sim 8 \pi \mathrm{mrad})$ for a narrow waist of the order of one wavelength and the ability to self reconstruct, which are the main signatures of diffraction-free beams. (c) 2013 Optical Society of America

OCIS codes: $240.6680,250.5403,050.1940$.
\end{abstract}

The unique properties of surface plasmon polaritons (SPPs), such as subwavelength confinement and surface field enhancement, have led to intensive investigations (both fundamental and applied) in this field, commonly known as plasmonics, with a significant portion being devoted to the design and fabrication of micro- and nano-optical components for SPP manipulation [1]. Although SPPs can be confined to relatively small transverse regions, using for example dielectric-loaded SPP waveguides [2], the inherent increase of effective index results in a decrease of propagation length. On the other hand, SPP beams that propagate along unstructured metal surfaces exhibit generally a longer propagation length, but exhibit weak localization and diverge quickly. A possible solution to overcome this tradeoff between confinement and propagation could be the generation of diffraction-free plasmonic beams (DF-SPPBs).

Diffraction-free beams are characterized by a field distribution, whose transverse intensity profile is invariant along the propagation direction and has the ability to reconstruct itself if an obstacle is placed along the propagation axis. At the same time, the beam waist can be rather small, i.e., of the order of one wavelength. Such an ideal beam cannot be realized experimentally, since it requires infinite energy and an infinite spatial extent. Nevertheless, quasi-diffraction-free beams can be generated to exist in a spatially limited region. Durnin et al. [3] ] studied for the first time these kinds of beams, namely, the Bessel beams. In recent years, other diffraction-free beams, such as Mathieu [ $\underline{4}$ ] and Airy beams []ㅡ have also been explored.

The above-mentioned beams are solutions to the freespace three-dimensional Helmholtz equation (no boundaries, guiding surfaces, or nonlinear media). Therefore, the very existence of two-dimensional (2D) diffractionfree solutions is not straightforwardly warranted. In fact, it was shown that the Airy beam is the only possible diffraction-free solution to the 2D paraxial wave equation [].

In this context, plasmonic (SPP-based) Airy beams have been studied theoretically $[\underline{7}, \underline{8}]$ and demonstrated experimentally [ㅁ-12]. At the same time, recent numerical studies in photonic crystals [토] demonstrated that diffraction can be tailored to generate $2 \mathrm{D}$ diffractionfree beams that resemble the profile of a Bessel function and present the self-healing property. Furthermore, a similar numerical approach was employed to model the generation of DF-SPPBs with axicon-shaped arrays of metal nanoparticles [14].

In this Letter, we propose and demonstrate experimentally two simple structures for the generation of DFSPPBs utilizing the SPP excitation by metal ridges illuminated at normal incidence [14]. The intensity profiles of the generated DF-SPPBs closely resemble the zeroth- and first-order Bessel functions of the first kind. Moreover, the generated beams exhibit the selfhealing ability, satisfactory beam localization, and a narrow beam-waist of the order of one wavelength.

Two ridge structures, $\mathrm{A} 1$ and $\mathrm{A} 2$, were fabricated on a $50 \mathrm{~nm}$ thin gold film supported by a glass substrate in order to generate two different kinds of DF-SPPBs targeting the excitation wavelength of $700 \mathrm{~nm}$. Structure A1 consists of two gold ridges (300 nm wide, $70 \mathrm{~nm}$ thick, and $15 \mu \mathrm{m}$ long, each) joined at one of their ends forming an angle $\theta$ [Figs. 1(a) and 1(b)]. Structure A2 is basically the same, but the ridges are shifted apart by half of the design SPP wavelength [Fig. 1(c)]. The idea of choosing these geometries was inspired by the axicon configuration [3] and nanoparticle arrays studied theoretically [14]. Our structures, unlike the latter one [14], were configured to produce Bessel-like beams upon conversion of an incident free propagating radiation into a SPP beam. Structures A1 and A2 were designed to generate beams with the zeroth- and first-order Bessel intensity profiles, respectively. The ridges were fabricated using a common procedure of electron-beam lithography followed by liftoff [Fig. 1(d)]. A $0.17 \mathrm{~mm}$ thin glass cover slip was used as a substrate to hold the sample.

The structures were illuminated at normal incidence with a weakly focused laser beam covering the whole structure at a wavelength $\lambda=700 \mathrm{~nm}$. The images were recorded in a charge-coupled device (CCD) camera using leakage radiation microscopy (LRM). The interference of the excited SPPs generates the DF-SPPB at the side of the 

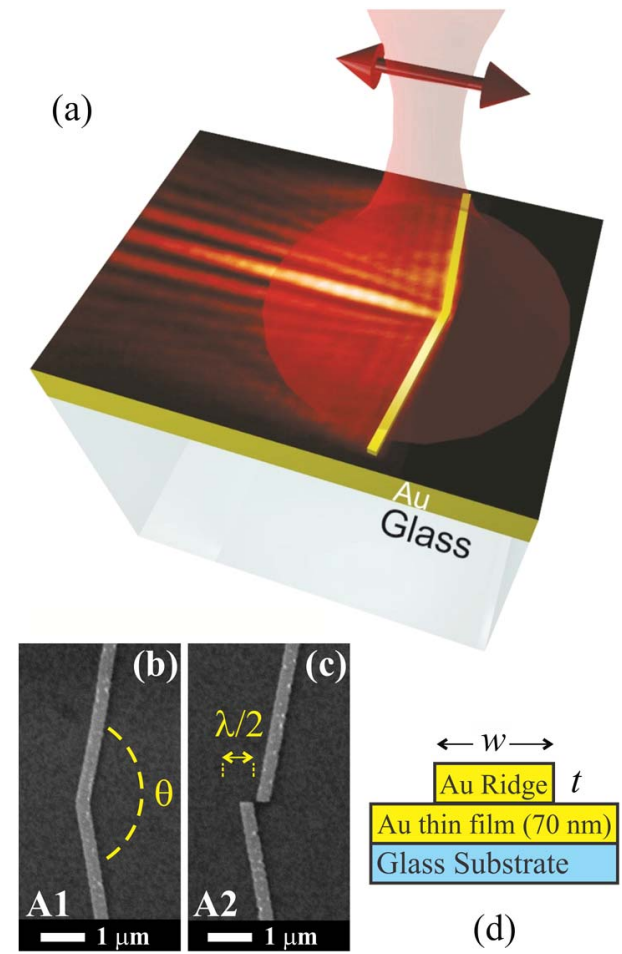

Fig. 1. (Color online) (a) Weakly focused laser beam illuminates a gold ridge structure to generate a DF-SPPB. The arrow indicates the polarization direction. (b) Scanning electron microscopy (SEM) image of the plasmonic axicon-like structure A1. (c) SEM image of the phase-shifting plasmonic axicon-like structure A2. (d) Cross-sectional schematic view of structures $\mathrm{A} 1$ and A2. The width $w$ and thickness $t$ of the gold ridges are 300 and $70 \mathrm{~nm}$, respectively.

structure where the angle $\theta$ is formed. SPPs are also excited in the opposite direction, forming two diverging beams, but since we are not interested in these contributions, we filtered them out by placing spatial filters in the $\mathrm{k}$-vector space. The directly transmitted light was also blocked to avoid saturation. For structure A1, the LRM image shows a well-localized beam centered along the $x$-axis with a clear correspondence to the zeroth-order Bessel beam [Fig. 2(a)]. The LRM image from structure A2 [Fig. 2(b)] clearly depicts a hollow beam that is characteristic of higher-order Bessel beams. In both cases, the generated beams exhibit a negligible divergence $(\sim 8 \pi \mathrm{mrad})$ and a full width at half-maximum (FWHM) of $1.1 \mu \mathrm{m}(\sim 1.5 \lambda)$ at the point where the beam intensity has its maximum value [Figs. $2(\mathrm{c})$ and $2(\mathrm{~d})]$ ]. The generated beams show intensity profiles with good correspondence to squared zeroth- and first-order Bessel functions of the first kind [Figs. 2(e) and 2(f)]. In order to generate the DF-SPPBs, it was necessary to illuminate the complete structure. The generated beams are a result of the interference of the SPP beams that are launched from each ridge, and thus a larger beam diameter reinforces their construction. Moreover, it was found that a better focused beam (waist of $\sim 5 \mu \mathrm{m}$ ) produces SPP beams with a higher divergence and broader waists [Fig. 2(g)]. The laser was also focused on a straight ridge to compare the divergence of usual SPP beams with the ones generated with the axicon-like structures [inset in
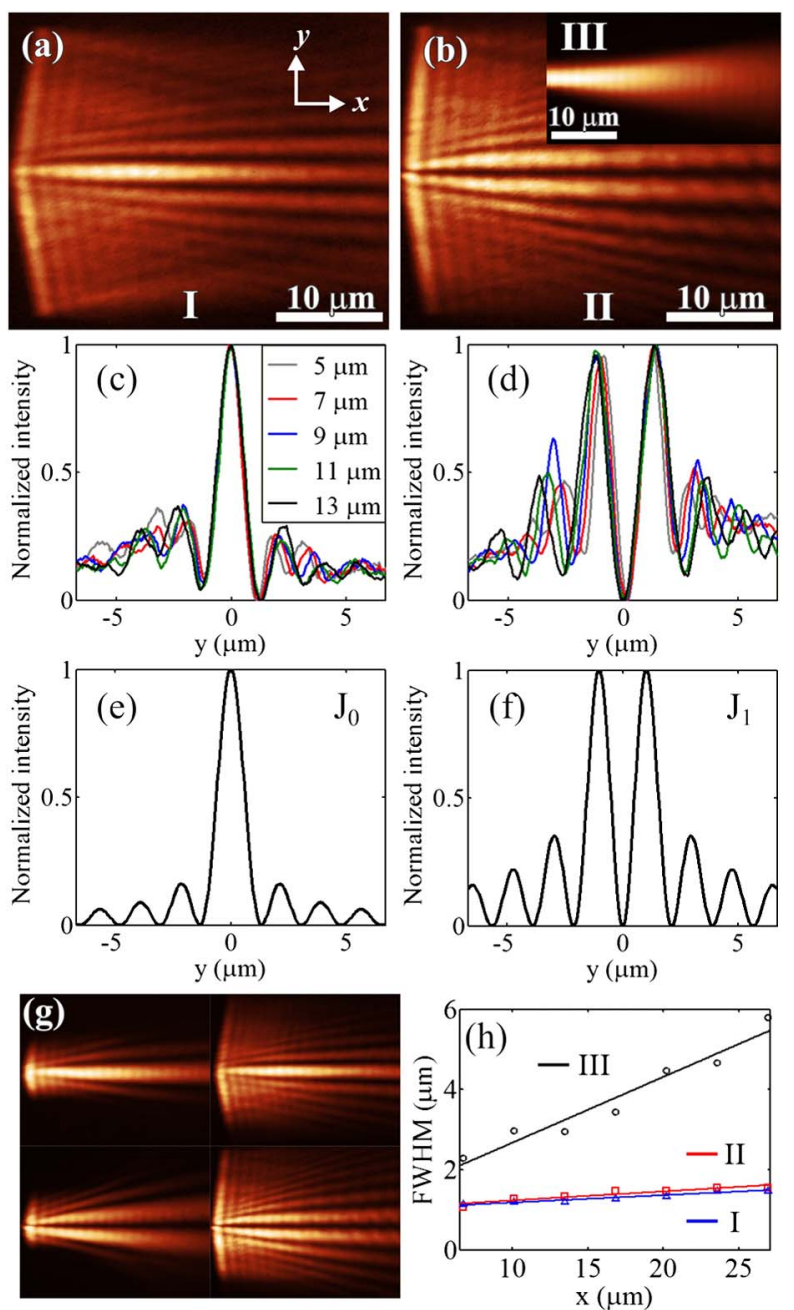

Fig. 2. (Color online) LRM images of DF-SPPBs generated by structure (a) A1 and (b) A2. The inset in (b) is the LRM image of a SPP beam excited with a straight ridge. (c) and (d) Averaged cross-sections along the $y$-coordinate at different distances from the apex (origin) of $\mathrm{A} 1$ and $\mathrm{A} 2$, respectively, to be compared with (e) zeroth- and (f) first-order squared Bessel functions. (g) LRM images of DF-SPPBs generated by illuminating the structure with a tightly focused beam $(\sim 5 \mu \mathrm{m})$ (left) and partially defocused $(\sim 10 \mu \mathrm{m})$ (right). (h) FWHM measurements at different distances from the apex (origin) for the three different beams labelled as I, II, and III in (a) and (b).

Fig. 2(b)]. It was found that the divergence of the SPP beam launched with the straight ridge was larger by one order of magnitude ( $\sim 80 \pi \mathrm{mrad})$ even though its width was larger [(Fig. 2(h)].

A second set of structures was fabricated adding a cylindrical gold bump structure placed at $10 \mu \mathrm{m}$ from the apex of the axicon-like structure (A1) using the same fabrication method mentioned before [Figs. 3(a) and 3(b)]. The radius $R$ and thickness $t$ of the cylinder were 150 and $70 \mathrm{~nm}$, respectively. The purpose of fabricating this structure was to insert an obstacle in the optical path of the DF-SPPB and observe if the generated beam is able to reconstruct itself. The LRM image of the DF-SPPB generated by illuminating structure A1 shows that the central beam remains unaltered in the presence of the obstacle [Fig. 3(c)]. Moreover, the main features of the intensity 


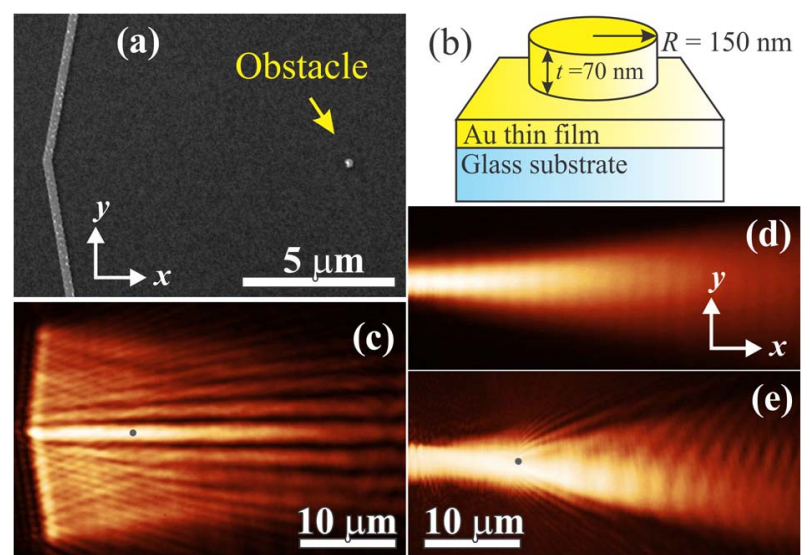

Fig. 3. (Color online) (a) SEM image of the axicon-like structure and a cylindrical obstacle placed at $10 \mu \mathrm{m}$ from apex of the structure. (b) Schematic design of the fabricated obstacle in (a). (c) LRM image of the generated DF-SPPB facing a cylindrical obstacle. LRM image of a typical SPP beam excited by focusing the laser beam on a straight ridge (d) propagating freely and (e) facing a cylindrical obstacle. The grey spot in (c) and (e) shows the position of the obstacle.

distribution are essentially the same as the one without the obstacle [Fig. 2(a)].

The cylindrical obstacle was also placed in front of a straight ridge to compare the perturbation induced in common SPP beams. Typical interference fringes in the form of parabolas are present in the LRM images showing the effect of the obstacle [Figs. 3(d) and 3(e)].

In conclusion, we have proposed and experimentally demonstrated the generation of DF-SPPBs by axiconlike structures that mimic the functionality of an axicon. In analogy with optical axicons, it was possible to generate limited-diffraction plasmonic beams with the zeroth-order Bessel intensity profiles within a limited region. Additionally, a variation of these structures allowed the generation of diffraction-free hollow beams with close resemblance to higher-order Bessel functions, specifically, the first-order Bessel functions. The generated DF-SPPBs showed a narrow waist $(\sim 1.5 \lambda)$ of less than two wavelengths. The self-healing ability, that is common in diffraction-free beams, was also satisfied, opening an alternative to use the generated DF-SPPBs, for example, for plasmonic tweezers [15].
Note added in proof: When completing our work, we have learned of a similar approach being reported [16] and concerned with the generation of cosine-Gauss SPP beams.

Garcia-Ortiz and Coello acknowledge the support given by Facultad de Ciencias Físico-Matemáticas, scholarship 228959 and project 127589 from CONACYT. Bozhevolnyi acknowledges the financial support from the Danish Council for Independent Research (the FTP project ANAP, Contract No. 09-072949). Han acknowledges the support from the National Natural Science Foundation of China (Grant No. 61107042).

\section{References}

1. T. Ebbesen, C. Genet, and S. I. Bozhevolnyi, Phys. Today 61(5), 44 (2008).

2. C. Garcia, V. Coello, Z. Han, I. P. Radko, and S. I. Bozhevolnyi, Appl. Phys. B 107, 401 (2012).

3. J. Durnin, J. J. Miceli, Jr., and J. H. Eberly, Phys. Rev. Lett. 58, 1499 (1987).

4. J. C. Gutiérrez-Vega, M. D. Iturbe-Castillo, and S. Chávez-Cerda, Opt. Lett. 25, 1493 (2000).

5. G. A. Siviloglou, J. Broky, A. Dogariu, and D. N. Christodoulides, Phys. Rev. Lett. 99, 213901 (2007).

6. G. A. Siviloglou and D. N. Christodoulides, Opt. Lett. 32, 979 (2007).

7. A. Salandrino and D. N. Christodoulides, Opt. Lett. 35, 2082 (2010).

8. W. Liu, D. N. Neshev, I. V. Shadrivov, A. E. Miroshnichenko, and Y. S. Kivshar, Opt. Lett. 36, 1164 (2011).

9. P. Zhang, S. Wang, Y. Liu, X. Yin, C. Lu, Z. Chen, and X. Zhang, Opt. Lett. 36, 3191 (2011).

10. A. E. Klein, A. Minovich, M. Steinert, N. Janunts, A. Tünnermann, D. N. Neshev, Y. S. Kivshar, and T. Pertsch, Opt. Lett. 37, 3402 (2012).

11. L. Li, T. Li, S. M. Wang, C. Zhang, and S. N. Zhu, Phys. Rev. Lett. 107, 126804 (2011).

12. A. Minovich, A. E. Klein, N. Janunts, T. Pertsch, D. N. Neshev, and Y. S. Kivshar, Phys. Rev. Lett. 107, 116802 (2011).

13. H. Kurt and M. Turduev, J. Opt. Soc. Am. B 29, 1245 (2012).

14. I. P. Radko, V. S. Volkov, J. Beermann, A. B. Evlyukhin, T. Søndergaard, A. Boltasseva, and S. I. Bozhevolnyi, Laser Photon. Rev. 3, 575 (2009).

15. M. L. Juan, M. Righini, and R. Quidant, Nat. Photonics 5, 349 (2011).

16. I. Lin, J. Dellinger, P. Genevet, B. Gluzel, F. de Fornel, and F. Capasso, Phys. Rev. Lett. 109, 093904 (2012). 